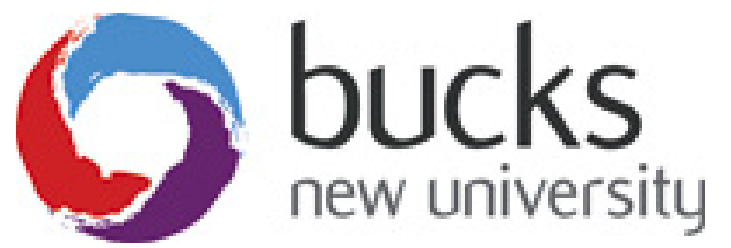

This is an Accepted Manuscript of an article published by RCNi in Nursing Management on 25 January 2018.

The final published version is available at: http://dx.doi.org/10.7748/nm.2018.e1674 


\title{
Simulation in a variety of settings using 'live' adult patient Service Users and moulage
}

\begin{abstract}
This article shows how simulation can be modified and adapted to benefit Higher Education Institutes (HEIs) in a variety of situations. These situations include the engagement of Service Users and moulage to ensure skill enhancement so the very best student experience can be harnessed empowering and supporting students in learning, as a means to achieving their potential.
\end{abstract}

This article describes a unique collaboration between one Higher Education Institute and Service Users (SUs), who have worked together in engaging in simulation activities in partnership. The development of simulation using Service Users is at the heart of developing student nurse education and recruitment. It has created communities of practice working across the traditional departmental boundaries to create innovative and creative learning opportunities for students.

This collaboration links to the political imperative to improve the education of the health and social care sector, highlights the integration of skills development to theory, focuses on person-centred care and demonstrates how this $\mathrm{HEl}$ is producing a compassionate and caring workforce.

Keywords: Simulation, Service Users, Moulage,

\section{Introduction}

This work on using SUs in 'live' human simulation with moulage encapsulates the breadth, creativity and innovation required to develop the necessary level of competence required for the future nursing workforce. The use of 'live' simulation can enhance the student experience, as it emphasises student engagement and involvement in and application to their own practice.

The breadth of the simulation activities brings together educators' with other stakeholders e.g. SUs and provides an approach towards educational developments that serve to improve the student experience and learning. It provides a safe environment for students to learn and grounds their learning in 'real life'.

\section{Simulation}

In recent years simulation has exploded into healthcare and nursing education. Its use has enabled educators to create experiences that simulate the clinical setting. Simulated practice creates a safe non-judgmental arena for students to learn and make mistakes with no harm to the patient. Thus, enabling the student to gain valuable knowledge through experiential learning. 
Nickles (2011) states that the inclusion of simulation has been lauded as able to address care skills deficits in health care education. Warland (2011) adds that simulation is considered as a means to promote a variety of health care students' learning of work organisation and people management skills. Hope et al. (2011) condenses the many advocates of using simulation in healthcare education by purporting that it enhances the acquisition of clinical skills in preparation for practice. Thus, Nickles (2011), Warland (2011) and Hope et al. (2011) all advocate the important role of simulation in a variety of health care courses in higher education. A view that is supported by McCaughey and Traynor (2010) and McCallum (2007) who are currently also in favour of using simulation in health care education. In addition, there is good evidence to demonstrate effectiveness of simulation in nursing (Alinier, 2006; Goldenburg et al., 2005; Nunn, 2004).

There is increasing evidence (Moule, et al., 2008; Motola, et al., 2013) that students undertaking health care courses want more simulation. Baxter et al., (2009) alludes to the idea that students want more simulation, not just to develop competency in clinical skills performance, but learning about how all the information they are acquiring throughout their course all fit together in the holistic practice of caring for individual patients. According to Kardong-Edgren et al. (2012) most nursing simulations follow a similar design, and rarely goes outside of the traditional cardiopulmonary resuscitation/deteriorating patient style set up. From the author's experience this idea continues and applies to current health care simulation set up.

In this HEI we provide pre-registration nursing students with medium and high fidelity manikins. In addition, attempt to offer a realistic clinical environment with the addition of contemporary authentic scenarios (students and practitioners own stories from practice and service user stories of their experiences) in their simulation sessions. There is the added advantage of video and audio facilities for self and peer review and debrief.

However, despite the use of a variety of learning and teaching aids in simulation, student evaluations following the use of medium fidelity manikins, a common theme emerged regarding their use of 'dummies'. Students felt they could perform better with a real person. Students' evaluations stated that: "It's hard to stay focused when simulating practice on a dummy, having real people would help in being practical and wanting to contribute knowledge and information."

"The dummy's used for simulation are not life-like. There are things such as, capillary refill that cannot be performed. This would be sorted out if we had an actual human to practice on."

"It will be nice to have real life patients as it will help to understand the case studies in more depth."

In their feedback students identify the 'dummies' as effecting and reducing their ability to perform well. The students imply nurse educators interpret this hindrance to their performance in some way as a lack of competence in their ability to practice at the bedside. Yet the main failure of simulation equipment is that it neglects to maintain humanity amidst potentially objective depersonalised high and medium fidelity technology. Simulation has its place 
but appears that it is not a substitute for a real person, as alongside skills application at the bedside interpersonal and consultation skills are essential.

\section{Service Users in nurse education}

There is a growing body of evidence to suggest that SU involvement in healthcare is beneficial (DoH, 2004; Jones, 2006; Nazarjuk, et al., 2013). According to McKeown et al. (2012) SUs and carer involvement in health and social care education has grown over recent years, and the NMC (2010) and GMC (2009) strongly recommend and advocate their use for nurse and medical education. However, according to Bollard et al. (2012) the involvement of SUs in adult nursing education is rare.

The Nursing and Midwifery Council (NMC) state in their Standards for Preregistration Education (NMC, 2010) that it is mandatory for HEls to demonstrate at all levels engagement with service users. The SUs are part of a partnership with the University, which has been growing over a number of years. The SUs are part of a larger organisation known as 'Independent voices'. The SUs are volunteers who all have had experiences (good and bad) of healthcare services. In our institution SUs are incorporated in a number of areas to enhance the student experience.

\section{Curriculum management}

For many years now in accordance with the NMC the university have been working with service users as part of curriculum management. SUs are part of all our current pre-registration programme committees. These committees meet twice per year and are comprised of programme and module leaders, students, administrators and SUs. The purpose of these committees is to regularly review the nursing programme.

\section{Development of student interpersonal skills}

There has been an increase in SU activity in classroom settings. Lecturers are increasingly asking for SU participation in sessions such as discussing their experiences or health care journeys, so students can appreciate some of the wider issues involved in delivering care. Prior to these sessions, the requesting lecturer briefs the $S U$ on session requirements and level of the student group. They are asked if there are any questions or topics that are "off-limits" and the students are also briefed accordingly, prior to the session. These situations provide a means to promote nursing students' learning about communication, and written feedback and university module evaluations have been very positive. This enables students to take this learning into real-life ward situations.

\section{Development of nursing skills}

Students have been engaged with SUs as part of their learning in other areas. Student nurses perform non-invasive procedures such as vital signs measurements or infection control practices. This helps students develop their 
confidence and communication skills, as well as practice at actual procedures. This ensures students' get the chance to put into practice what they have learned in the classroom and maximizes their learning opportunities, and has been very well evaluated.

In 'live' simulation

This involves the use of human patient simulations, whereby SUs are employed to act as patients, doctors or relatives, bystanders, helpers. The use of SU involvement in simulation provides students with the opportunity to gain a clear picture of practice, whereby there is time to think critically and make decisions in a safe environment (Photos $1 \& 2$ ).

The inclusion of and input from SUs into the education of students is timely and responsive to current research and literature, but also used here is shown to be effective and an important part of student learning. Student feedback states:

"I found this very helpful and helped me to think about how I plan my care for more than one patient at a time while helping other staff on the ward" "It reminds me of the actual ward with real patients."

"Real patients keep you updated about their feelings and current condition" "The service users were very realistic and definitely helped us to carry out our assessment"

The addition of 'live' simulations shows to students the potential vulnerability of patients and how our HEI is educating students, who obtain a professional qualification, to be compassionate and caring and highlights that this issue receives serious attention. Additional themed 'live' simulation feedback can be viewed in Table 1.

'Live' simulation mock ups using SUs in the skills laboratory include, caring for individual patients at the bedside, a clinical ward set up, wound management, infection control practices, and aseptic technique. The 'live' simulations include self and peer assessment between student groups using a paper exercise with students at the bedside and video playback. This learning is overseen to ensure accuracy and this continues through to debrief (both for SUs and students), which is a key learning element, to ensure both educational development and emotional support is delivered in a safe environment.

The 'live' simulations with SUs provide students with the opportunity to think critically, make decisions and develop reflection-in-action skills. The 'live' simulations can serve to prepare students for the professional work environment. This can help facilitate student learning about real live situations they will encounter in clinical practice.

On taster days/ open days for new applicants

The idea of 'live' human simulations using service users, technology based manikins with the addition of moulage is also used at interview and taster 
days for potential candidates looking to undertake a degree on one of our health related courses (Photos $3 \& 4$ ).

\section{Provide feedback}

SUs really enjoy being involved in the simulation aspect of their activity and respond well to students. They give feedback there and then to students on their performance, particularly in relation to their personal traits, communication skills and essential nursing skills. SU feedback to students state:

"Due to nervousness, some students were a little lost, others flourished" "The student introductions were good, showed empathy"

For additional SU feedback see Table 2. This immediate feedback is very much appreciated by students. In addition, SUs provide feedback to the lecturers on the process and set up of the simulation, which is used to influence developments (Table 3).

Provide study-days to help SUs develop their skills

SUs who are used regularly by the university are invited to attend up-date sessions to help develop their abilities to play the role of a variety of different types of patients other than themselves. YouTube clips are accessed to identify common conditions that bring patients into hospital e.g. COPD, asthma and these are shown to the SUs. Some scenarios are provided for SUs to act out in a skills simulation situation and these are videoed and played back. Evaluations of the up-date sessions have been very favorable:

"How to be a more genuine patient with enough acting to be a real patient for the nurses' experience.

"Learnt the extent to which we can role-play. The challenges faced by nurses and also relatives. Better understanding of 3 illnesses from the videos"

All SUs requested more of same and additional development e.g. in wanting a session on providing verbal feedback to students (see Table 4 for more feedback comments from SUs).

\section{Moulage}

Moulage is the application of casualty make-up for training purposes and to simulate clinical presentations (Pywell, et al., 2016). The skills and the authenticity in a simulation is a key essential to the psychological buy-in of students (Smith-Stoner 2011). The use of moulage with simulation is a creative direction to develop skills, which adds authenticity.

The creation of moulage can vary in application and time taken to apply. For example, made up wounds can be devised using specialist make-up, which is available to purchase, however for sessions using moulage to be sustainable, workable and successful the cost has to be minimal, and many of the wounds are created using inexpensive household ingredients. The use of such materials allows the creation of authentic looking skin tissue, which includes the appearance of slough, redness, swelling, the presence of pus, which gives 
a clue to a possible wound infection, all adding to the authenticity of simulation.

By adding moulage wounds, body fluids and bowels to scenarios we add legitimacy to the scene. The various moulage currently used can be seen in Table 5. One particular moulage used with SUs is wound simulation; this can be just the wound or integrated as part of daily management and care.

Wound moulage

In simulated skills sessions usually to identify a wound the traditional use of photographs are used for students to look at and assess. This method is defined as low fidelity two-dimensional simulated practice. Moulage was introduced to simulation manikins to facilitate nurses to improve their understanding of wound assessment, dressings and infection control practices, and how it fits into every day nursing care. This provides a threedimensional view of wounds and raises the simulation session to mid-fidelity.

The addition of wound moulage to manikins helps develop student understanding of the aetiology and physiology of wounds and how they heal. Samur (2014) outlines that effective wound assessment and management is a key clinical skill required for any practitioner to successfully improve tissue healing and prevent further breakdown. This information plus the knowledge of dressings and how they interact with the wound becomes meaningful through the addition of moulage, thus ensuring best practice treatments are provided at the bedside (Photos $5 \& 6$ ).

\section{Moulage with Service Users}

With the introduction of SUs in 'live' simulation alternative methods to photographs and wound moulage on manikins was introduced to incorporate wound moulage to SUs.

Smith-Stoner (2011) asserts that the addition of wound moulage to SUs rather than manikins is that it can enhance the authenticity of simulation. According to Merica (2012) a benefit of using moulage with SUs is that the wound is blended into the SUs skin, making it appear like a real wound. All of these give legitimacy to the scene and are created for students to examine and report their findings.

This mid fidelity three-dimensional wound experience with moulage and SUs, for the students, enables them to closely inspect the wound. Students have the opportunity to look at a 3D example, they are able to really inspect the wound moulage, and discuss with the patient (SU) about their wound e.g. pain, discomfort, smell adding a psychological dimension to wound care. In addition, decide appropriate assessment and interventions. Student feedback states:

"Made the learning real"

"It improves the practical learning experience to be transferred to clinical areas" 
"Enhanced my skills in wound dressing as I don't encounter all types of wounds in my work"

The addition of moulage to 'live' simulation with SUs has been engaging and shown to be of great benefit to students, as not all students are able to see all areas of care in their clinical practice, moulage has given them this opportunity. Additional student evaluations on how moulage and SU input has enhanced their learning experience can be viewed in Table 6 .

\section{Paying attention to detail}

The application of moulage has already added authenticity to 'live' simulation. A challenge to undertaking this work was to make the simulations more life like. This was addressed by complementing the moulage with 'real life' scenarios and the application of the appropriate props at the bedside e.g., by paying attention to detail. A number of studies suggest that attention to small details makes a big difference to the provision of compassionate care (Graber \& Mitcham, 2004; Perry, 2009 \& Van der Cingel, 2011). Paying attention to the 'little things' in respect to simulation is an attempt to create an environment whereby care and compassion can be demonstrated. The gathering of 'real life' scenarios and the preparation of additional teaching materials generated additional work, yet the benefits of witnessing total student engagement for student learning far outweighed any downside in collecting and preparing resources.

Paying attention to detail includes teaching materials such as doctors' notes, drug charts, fluid balance charts, vital signs (NEWS chart), VIP, blood results, X-Rays, wound/ pain assessments, and props such as wash bag, juice, water, clean pyjamas, and are all included to make simulations more like 'real life'.

Simulation with SUs and moulage is not only about students being able to assist a 'real life' patient with fundamental care or life saving interventions, it is about the attention given to creating a real looking environment, such as, the inclusion of 'real life' scenarios, teaching materials and other props.

\section{Conclusion}

The use of both medium and high fidelity manikins is very successful with the combination of 'real' human service users and moulage. The 'live' simulation with service users and moulage strives to prepare students for the professional work environment they will encounter in their later roles. It can impact on students' development enabling them to recognise and respond to clinical situations and emergencies. Attempts to demonstrate the importance of teaching clinical subjects in a way that connects practice to the academic side of the course bringing them both to life. In addition, serves as a means to develop useful skills, such as compassion, communication, care, competence and reflection-in-action (moment-to-moment decision-making skills), all of which are essential skills when caring for individual patients at the bedside. 


\section{References}

Alinier, G., Hunt, B., Gordon, R., Harwood, C. (2006) Effectiveness of intermediate fidelity simulation training technology in undergraduate nursing education, Journal of Advanced Nursing, 54(3), 359-369

Baxter, P., Akhtar-Danesh, N., Valaitis, R., Stanyon, W. and Sproul, S. (2009) Simulated experiences: Nursing students share their perspectives, Nurse Education Today, 29, 859-866

Bollard, M., Lahiff, J., Parker, N. (2012) Involving people with learning disabilities in nurse education: towards an inclusive approach, Nurse Education Today, 32(2), 173-177

Department of Health $(2004,2007)$ Patient and public involvement in health, London: $\mathrm{DH}$

General Medical Council (2009) Supplementary advice to tomorrow's doctors: patient and public involvement in undergraduate medical education, www.gmc-uk.org/education/undergraduate/8837.asp

Goldenburg, D., Andrusyszyn, M. A., Iwasiw, C. (2005) The effect of classroom simulation on nursing students' self-efficacy related to health teaching, Journal of Nurse Education, 44(7), 310-314

Graber, D. R., Mitcham, M. D. (2004) Compassionate clinicians take patient care beyond the ordinary, Holistic Nursing Practice, 18(2), 87-94

Hope, A., Garside, J. and Prescott, S. (2011) Rethinking theory and practice: Pre-registration student nurses experiences of simulation teaching and learning in the acquisition of clinical skills in preparation for practice, Nurse Education Today, 31, 711-715

Ironside, P. M. (2004) "Covering content" and teaching thinking: deconstructing the additive curriculum, Journal of Nursing Education, 43(1), 512

Jones, C. (2006) Involving NHS Service Users in teaching advanced clinical skills, British Journal of Nursing, 15(8), 462-465

Kardong-Edgren, S., Oermann, M., Odom-Maryon, T. (2012) Challenges in performing CPR: Implications for RNs, Journal of Nurses in Staff Development, 28(1), 9-15

McCallum, J. (2007) The debate in favour of using simulation education in pre-registration adult nursing, Nurse Education Today, 27, 825-831

McCaughey, C. S. and Traynor, M. K. (2010) The role of simulation in nurse education, Nurse Education Today, 30, 827-832 
McKeown, M., Milihi-Shoja, L., Hogarth, R., et al. (2012) The value of involvement from the perspective of service users and carers engaged in practitioner education: not just a cash nexus, Nurse Education Today, 32(2), 178-184

Merica, B. J. (2012) Medical moulage: How to make your simulations come alive, Philadelphia: Davis Company

Motola, I., Devine, L. A., Chung, H. S., Sullivan, J. E., Issenburg, B. (2013) Simulation in healthcare education: A best evidence practical guide. AMEE Guide No. 82, Medical Teacher online http://www.tandfonline.com/loi/imte20

Moule, P., Walford, A., Sales, R., Lockyer, L. (2008) Student experiences and mentor views of the use of simulation for learning, Nurse Education Today, 28(7), 790-797

Nazarjuk, A., Burnal, C., Southgate, A. (2013) Involving service users in student education, Learning Disability Practice, 16(5), 14-19

Nickles, L. J. (2011) The use of simulation to address the acute care skills deficit in pre-registration nursing students: A clinical skills perspective, Nurse Education in Practice, 11, 199-205

Nunn, A. (2004) Almost the real thing, Nursing Management, 11(7), 14-18

Nursing and Midwifery Council (2010) Standards for pre-registration nursing education, tinyurl.com/6zodwq9

Perry, B. (2009) Conveying compassion through attention to the essential ordinary, Nursing Older People, 21(6), 14-21.

Pywell, M. J., Evgeniou, E., Highway, K., Pitt, E., Estela C. M. (2016) High fidelity, low cost moulage as a valid simulation tool to improve burns education, Ovid Emcare Burns, 42 (4) 844-852 http://dx.doi.org/10.1016/j.burns.2015.12.013

Samur, S. (2014) I, Patient: Performance Practices in Medical Simulation at Hôpital Montfort, Canadian Theatre Review, 159(1), 52-57

Smith-Stoner, M. (2011) Using moulage to enhance educational instruction, Nurse Educator, 36(1), 21-24.

Van der Cingel, M. (2009) Compassion and professional care: exploring the domain, Nursing Philosophy, 10, 124-136.

Warland, J. (2011) Using simulation to promote nursing students' learning of work organisation and people management skills: A case study, Nurse Education in Practice, 11, 186-191 